https://doi.org/10.23913/ride.v12i23.1058

Artículos científicos

\title{
La promoción de la lectura en la vinculación universitaria
}

\author{
The promotion of reading in the university link
}

\section{A promoção da leitura no vínculo universitário}

\author{
Antonia Olivia Jarvio Fernández \\ Universidad Veracruzana, México \\ ojarvio@uv.mx \\ https://orcid.org/000-0003-2350-0289
}

\section{Resumen}

Como respuesta a las exigencias globales, las instituciones de educación superior han asumido un nuevo paradigma para la educación de calidad: la formación integral de ciudadanos con competencias básicas y profesionales sustentadas en valores humanistas, en el diálogo igualitario e inclusivo; es decir, formados en programas educativos altamente vinculados a los problemas sociales. La lectura y la escritura habilitan para aprender a lo largo de la vida y para neutralizar desigualdades de diverso tipo, por lo que la formación de lectores requiere de promotores profesionales que, con los conocimientos adecuados, incidan directamente en la transformación social. Por tanto, el objetivo del presente trabajo es contribuir a la promoción de la lectura y al uso de metodologías para la evaluación y mejora de proyectos en el ámbito lector, por lo cual se analiza el impacto de un programa que hace énfasis en la vinculación universitaria y en las políticas públicas.

En concreto, se presenta una revisión sistemática de 54 proyectos de intervención en promoción de la lectura y la escritura con diversos tipos de grupos. Los proyectos se diseñaron e implementaron a partir del enfoque de aprendizaje basado en proyectos (ABP), con una perspectiva sociocultural, y en el marco de un programa de posgrado. Para la revisión se realizó una clasificación por ámbito de intervención y la discusión de resultados se alineó con una revisión de literatura. Los resultados demuestran el impacto del programa en los diversos grupos atendidos, que incluyó a diferentes ámbitos educativos, grupos sociales, algunos considerados marginales o 


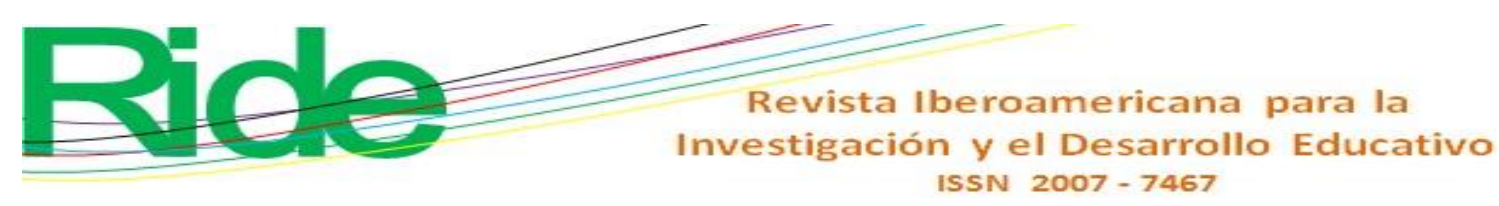

vulnerables. Se muestra un cambio en las percepciones sobre el significado de leer y el notable desarrollo en prácticas de la lectura y la escritura, así como en el de habilidades comunicativas. Se destaca la importancia del rol del promotor de la lectura en este proceso, con lo que se reafirma la importancia de su profesionalización como agente de cambio. El conocimiento obtenido permitirá retroalimentar las políticas institucionales. En este sentido, se identificaron los avances y los retos en la promoción de la lectura tanto para la institución como para la región, sobre la importancia de fortalecer los lazos comunicantes de la universidad con los sectores sociales.

Palabras clave: aprendizaje activo, educación superior, intervención, investigación participativa, lectura.

\section{Abstract}

In response to global demands, higher education institutions have assumed a new paradigm for quality education: comprehensive training of citizens with basic and professional skills, supported by humanistic values, in egalitarian and inclusive dialogue; that is to say, trained in educational programs highly linked to social problems. Reading and writing enable people to learn throughout their lives and to neutralize inequalities of various kinds, which is why the training of readers requires professional promoters who, with the appropriate knowledge, have a direct impact on social transformation. The objective of this work is to contribute to the promotion of reading, but also to the methodology for the evaluation and improvement of projects in this area, showing the impact of a program that emphasizes university ties and public policies.

A systematic review of 54 intervention projects to promote reading and writing with various types of groups is presented. The projects were designed and implemented based on the project-based learning approach $(\mathrm{PBL})$, with a sociocultural perspective, and within the framework of a graduate program. For the review, a classification was made by field of intervention and the discussion of results was aligned with a literature review. The results demonstrate the impact of the program on the various groups served, which included various educational settings, social groups, some considered marginal or vulnerable. It shows a change in perceptions about the meaning of reading and the remarkable development in reading and writing practices, as well as in communication skills. The importance of the role of the promoter of reading in this process is highlighted, thus reaffirming the importance of their professionalization as an agent of change. The knowledge obtained will allow feedback on institutional policies. In this sense, the advances and challenges 


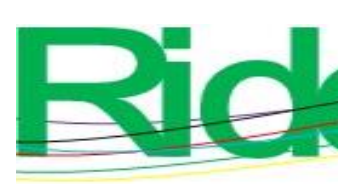

Revista Iberoamericana para la Investigación y el Desarrollo Educativo ISSN $2007-7467$

in promoting reading for both the institution and the region were identified and the importance of strengthening the communicating vessels of the university with the social sectors.

Keywords: active learning, higher education, intervention, participatory research, reading.

\section{Resumo}

Em resposta às demandas globais, as instituições de ensino superior assumiram um novo paradigma para uma educação de qualidade: a formação integral de cidadãos com competências básicas e profissionais amparadas em valores humanísticos, em um diálogo igualitário e inclusivo; ou seja, formados em programas educacionais altamente vinculados aos problemas sociais. A leitura e a escrita permitem que as pessoas aprendam ao longo da vida e neutralizem as desigualdades de vários tipos, razão pela qual a formação de leitores requer promotores profissionais que, com os conhecimentos adequados, tenham um impacto direto na transformação social. Portanto, o objetivo deste trabalho é contribuir para a promoção da leitura e o uso de metodologias de avaliação e aprimoramento de projetos na área da leitura, para os quais se analisa o impacto de um programa que privilegia a vinculação universitária e nas políticas públicas.

Especificamente, é apresentada uma revisão sistemática de 54 projetos de intervenção para promoção da leitura e escrita com vários tipos de grupos. Os projetos foram concebidos e implementados a partir da abordagem de aprendizagem baseada em projetos (PBL), com uma perspetiva sociocultural e no âmbito de um programa de pós-graduação. Para a revisão, foi realizada uma classificação por campo de intervenção e a discussão dos resultados alinhada a uma revisão da literatura. Os resultados demonstram o impacto do programa nos diversos grupos atendidos, que incluem diferentes contextos educacionais, grupos sociais, alguns considerados marginais ou vulneráveis. Mostra uma mudança nas percepções sobre o significado da leitura e o notável desenvolvimento nas práticas de leitura e escrita, bem como nas habilidades de comunicação. Ressalta-se a importância do papel do promotor da leitura nesse processo, reafirmando a importância da sua profissionalização como agente de mudança. Os conhecimentos obtidos permitirão retroalimentar as políticas institucionais. Nesse sentido, foram identificados os avanços e desafios da promoção da leitura para a instituição e para a região sobre a importância de fortalecer os laços comunicativos da universidade com os setores sociais.

Palavras-chave: aprendizagem ativa, ensino superior, intervenção, pesquisa participativa, leitura. 


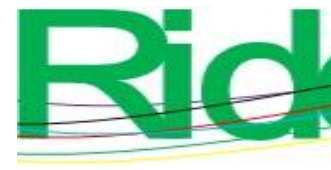

Fecha Recepción: Abril 2021
Revista Iberoamericana para la

Investigación y el Desarrollo Educativo ISSN 2007 - 7467

Fecha Aceptación: Octubre 2021

\section{Introducción}

La lectura y la escritura se han resignificado en los últimos años. Entidades como la Organización de las Naciones Unidas para la Educación (Unesco) y la Organización para la Cooperación y el Desarrollo Económicos (OCDE) resaltan su importancia para garantizar el aprendizaje significativo, ejercer la ciudadanía y enfrentar los retos del siglo XXI, de ahí que las políticas públicas de todos los países las asuman explícitamente. En tal sentido, el Centro Regional para el Fomento del Libro en América Latina y el Caribe (Cerlalc) (2011) ha evaluado periódicamente a los países de la región en cuanto a los programas empleados para el fomento de la lectura. En el caso de México, a pesar de las estrategias implementadas, las estadísticas de prácticas lectoras siguen señalando un reto pendiente, por lo que las instituciones educativas de todos los niveles se han replanteado la tarea de modificar las prácticas de enseñanza y los diseños curriculares.

Ahora bien, entre los programas de lectura desarrollados en México se pueden mencionar el de Salas de Lectura, creado en 1995 por el Consejo Nacional para la Cultura y las Artes (Conaculta). Este funciona en todo el país con voluntarios y atiende a distintos grupos sociales brindando asesoría y capacitación (Vizcarra, Ovalle y Corona Berking 2012). Luego, en 2001 aparece el primer Programa Nacional de Lectura (PNL) impulsado por la Secretaría de Educación Pública (SEP), junto con Libros del Rincón y el Programa Nacional para el Fortalecimiento de la Lectura y Escritura en la Educación Básica (Pronalees), los cuales distribuyeron colecciones de obras literarias e informativas en la educación básica del país, y capacitaron equipos técnicos en cada estado. Posteriormente, surge Hacia un país de lectores, derivado del PNL y la Estrategia Nacional 11+5, para una comunidad de lectores y escritores (SEP, 2012). Después, en 2020 se establece en el documento de la SEP Educación — dentro de las líneas estratégicas del programa Fortalecimiento de la Calidad Educativa - la Estrategia Nacional de Lectura, que integra tres ejes: carácter formativo, sociocultural e informativo. Además, como programa estratégico del gobierno, se está abordando la problemática con importantes apoyos económicos para su investigación e incidencia.

Sin embargo, cabe señalar que los resultados de los proyectos utilizados no ofrecen la información (o resulta muy complejo hallarla) sobre la evaluación para determinar el impacto en grupos específicos. En palabras de Kalman (2003), si bien es cierto que la distribución de acervos 


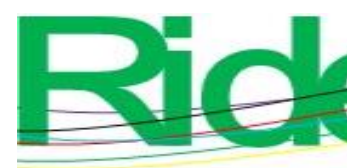

Revista Iberoamericana para la Investigación y el Desarrollo Educativo ISSN $2007-7467$

constituye una parte importante para promover la lectura, es "su circulación y uso entre las manos de los lectores lo que la fomenta" (p. 39).

En este contexto, México debe continuar trabajando en la construcción de una sociedad lectora, ya que a pesar de los esfuerzos realizados para tal propósito, solamente 3.5 libros son leídos al año por persona (Instituto Nacional de Estadística y Geografía [Inegi], 2019a), cifra que solo llega a 5 en el ámbito universitario, con un promedio de dos horas semanales dedicadas a la lectura, lo que refleja un déficit significativo.

Este panorama general ha ido empeorando, pues el porcentaje de personas lectoras de libros, revistas, periódicos, etc., ha disminuido $10 \%$ en comparación con las cifras de 2015. Adicionalmente, solo $25 \%$ de las personas entrevistadas acude a una sección de libros o revistas en tiendas departamentales, $20 \%$ a una librería y únicamente $11 \%$ a una biblioteca (Inegi, 2019a). Asimismo, se ha demostrado que a pesar del incremento en el uso de la tecnología, prevalece la lectura de materiales impresos.

En esta misma línea, y a través de PISA 2018, se encontró que los estudiantes mexicanos tienen serias dificultades en lectura, pues solo $1 \%$ alcanzó un desempeño sobresaliente en la prueba y más de la mitad se ubicaron en el nivel dos de competencia lectora (es decir, identificaron la idea principal de un texto de longitud moderada, encontraron información y reflexionaron sobre el propósito). En otras palabras, el desempeño del país entre los años 2000 y 2018 ha sido el mismo (OCDE, 2018), lo que, dadas las exigencias del milenio, se convierte en un retroceso.

Estos bajos índices demuestran que el proceso lector exige un acompañamiento que debe iniciar desde etapas tempranas, haciendo énfasis en el disfrute de los textos para agudizar la imaginación y ampliar el vocabulario y las habilidades metacognitivas. Una lectura más elaborada, por tanto, requiere del desarrollo de estrategias de comprensión y del reconocimiento de los elementos textuales para organizar y argumentar sobre el contenido. A partir de dar sentido al mensaje, se pueden conseguir inferencias e interpretaciones que obligan a plantearse nuevas preguntas a través de la capacidad de análisis y síntesis.

La materialización de este evento se visibiliza con la escritura como práctica para construir discursos ordenados, coherentes y con fuerza de contenido (Pardo, 2019; Sánchez y Errázuriz, 2018), lo que demanda constancia y continuidad a lo largo de la formación educativa, es decir, una nueva alfabetización — posterior a la alfabetización básica—. Por ello, la lectura y la escritura se asumen como prácticas permanentes, que adquieren características específicas de acuerdo con las etapas de la formación (Acosta y Pedraza, 2019; Escobar, Garbarini y López D’Amato, 2019). 


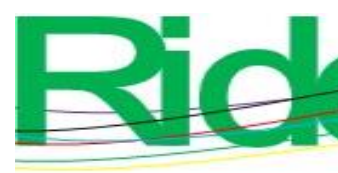

Revista Iberoamericana para la Investigación y el Desarrollo Educativo ISSN $2007-7467$

En palabras de Castro Azuara y Sánchez Camargo (2018), "llevó mucho tiempo reconocer (...) que se requiere más que cursos de ortografía y sintaxis para acercar a los estudiantes universitarios a los requerimientos y exigencias de la interacción comunicativa especializada" (p. 139). En efecto, además del dominio de reglas idiomáticas que se adquieren durante periodos largos y ejercicios constantes, se necesita del manejo de términos especializados de cada disciplina, como prácticas inherentes al aprendizaje y a la generación de conocimiento (Carlino, 2005; Natale, 2013; Stagnaro, 2018). En este sentido, Pérez (2018) puntualiza lo siguiente:

[El estudiante universitario] requiere no solo del conocimiento general de saberes retórico-discursivos, sino de los modos de aplicación de los mismos según criterios, valoraciones y objetivos epistemológicos de cada disciplina (...). Resulta importante una enseñanza de la lectura que permita la integración de los aportes provistos tanto desde una cultura disciplinar particular como desde los estudios de las alfabetizaciones académicas (p. 68).

Aunado a lo anterior, se deben considerar las transformaciones tecnológicas, las cuales han dado origen a nuevos discursos y formas de comunicación que han obligado a una preparación particular definida como alfabetización digital (Cassany, 2012; Cordón García, 2019; Pérez, 2018). Asimismo, se debe trabajar en el desarrollo de habilidades informativas -alfabetización informacional - para acercarse a los textos de la mejor manera posible, lo cual constituye un proceso de multialfabetización del siglo XXI (Martos Núñez y Campos Fernández-Figares, 2013).

Las instituciones, por ende, deben dar soporte al proceso de re-alfabetizar según las necesidades específicas, lo cual solo se puede conseguir formando agentes profesionales que incidan en dicho proceso. Esta alfabetización incluye la búsqueda y uso eficiente de la información (Cassany y Vázquez, 2014; Jarvio Fernández, 2019; Salado, et al., 2017) en diversos soportes, sin omitir las convenciones discursivas para la elaboración de los textos académicos de cada disciplina (Cordón García, 2019; Escobar et al., 2019).

La lectura, indispensable en el ámbito académico, trasciende a la educación e impacta en las esferas sociales. Ramírez Leyva (2016) señala que de nada sirve que la sociedad disponga de abundante información si no se cuenta con capacidades de lectoescritura suficientemente desarrolladas. Por ello, se hace necesaria la profesionalización de la promoción de la lectura, entendida como un saber innovador orientado al desarrollo de competencias para atender las necesidades de re-alfabetizar, con el objetivo de incrementar la competencia lectora en la población. 


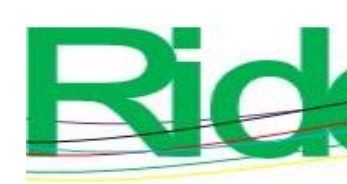

Revista Iberoamericana para la
Investigación y el Desarrollo Educativo
ISSN $2007-7467$

servicios bibliotecarios y de información y 49 bibliotecas; tiene una reconocida tradición editorial, así como en humanidades y cultura.

La vinculación en la UV como actividad estratégica garantiza la pertinencia al adecuar las funciones sustantivas a los requerimientos regionales. Su impacto en los problemas sociales contribuye a mejorar las condiciones de vida de la población, así como a elevar la competitividad y productividad de las instituciones gubernamentales, privadas y civiles. En esta orientación, la promoción de la lectura, como eje transversal, integra grupos multidisciplinarios con los que se promueve el desarrollo de habilidades y competencias, así como de prácticas letradas situadas. Con el Programa Universitario de Formación de Lectores (PUFL), creado desde el año 2006, se ha establecido una serie de vasos comunicantes con la sociedad.

\section{Avances en la lectura dentro de la Universidad Veracruzana}

El PUFL es un programa que promueve la lectura, sobre todo de literatura, entre su comunidad. Se diseñó originalmente para promover la Colección Biblioteca del Universitario, que integra obras imprescindibles, seleccionadas por Sergio Pitol, premio Cervantes 2011 responsable del proyecto hasta el día de su fallecimiento, en 2018-. Actualmente, la colección tiene 72 títulos: un canon de literatura, humanismo y ciencia. El PUFL desarrolla talleres y círculos de lectura en todas las regiones y dependencias de la UV, así como capacitaciones sobre cómo abordar la lectura en el aula. Las acciones trascienden a la comunidad universitaria, pues también se atiende a diversos sectores sociales, como direcciones de cultura, instituciones educativas, dependencias gubernamentales, etc.

Ante esta expansión se elaboraron estudios diagnósticos, con el diseño y aplicación de instrumentos para monitorear y evaluar avances del programa, y se capacitó a profesores, estudiantes, bibliotecarios y trabajadores. De estas actividades derivaron líneas de investigación sobre lectura en la educación superior y lectura digital. En el año 2012 se capacitó a promotores de lectura entre la comunidad estudiantil. Así surgió la experiencia educativa Taller para promotores de lectura, que se ofrece dentro del plan de estudios de los programas de licenciatura, del Área de Formación y Elección Libre. En 2016 se sumó el curso Música y literatura, donde la música es tema central para acercarse a los textos. Durante el desarrollo del PUFL emergió la necesidad de especialistas en promoción de la lectura, por lo que se concretó el posgrado para profesionalizar esta actividad. Así nació la Especialización en Promoción de la Lectura (EPL), en 2014. 


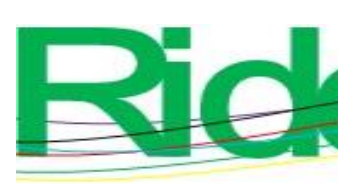

Revista Iberoamericana para la
Investigación y el Desarrollo Educativo
ISSN $2007-7467$

las prácticas de lectura y escritura de diversos grupos sociales, con un programa eficiente de promoción de la lectura?

\section{Hipótesis}

La profesionalización de promotores de lectura en la educación superior puede incidir en las prácticas de lectura y escritura de diversos grupos sociales, utilizando los métodos adecuados y los diferentes soportes de lectura, lo que redundará en el fortalecimiento de la vinculación universitaria, así como en el desarrollo de las políticas, planes y programas en este tema.

\section{Metodología}

Se realizó una revisión sistemática (Cooper, 2010) para identificar el impacto colectivo de las acciones de vinculación universitaria y para establecer los logros y retos en la promoción de la lectura. Se revisaron 54 reportes desarrollados con lineamientos y coordinación comunes de graduados de siete generaciones de la EPL (periodo 2014-2020). El enfoque metodológico de cada proyecto fue el de la investigación-acción (Romero y Barbosa, 2012), con una perspectiva sociocultural (Cassany, 2006), que inició con un diagnóstico del ámbito de intervención para determinar el problema, los objetivos particulares y las estrategias, así como la cartografía lectora por utilizar. Para la evaluación, cada proyecto empleó un enfoque mixto, a partir de evidencias como asistencia, participación, involucramiento y revisión de productos; asimismo, se consideraron expresiones y opiniones a partir de la técnica de observación participativa, utilizando bitácoras de campo y entrevistas semiestructuradas.

\section{Resultados}

Los 54 proyectos atendieron a 1149 participantes, y al menos $90 \%$ declararon haber recibido beneficios de las actividades expuestas. En $87 \%$ de los participantes se cambió la percepción de leer y la actividad se integró a su agenda personal. Asimismo, 9 de cada 10 declararon la importancia del promotor de la lectura como acompañante, y 5 de cada 10 compartieron sus lecturas con familiares y amigos.

La infografía de la figura 1 presenta los resultados más relevantes de la revisión sistemática de los reportes. Posteriormente, se presenta un análisis, síntesis y discusión, acorde a la tipología de los grupos de intervención. 


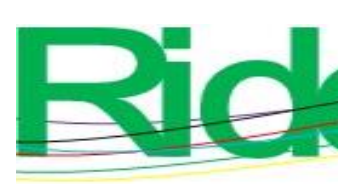

Revista Iberoamericana para la
Investigación y el Desarrollo Educativo
ISSN $2007-7467$

importancia del reconocimiento de los textos literarios en esta etapa para mejorar la expresión oral, la memoria, la atención y la comprensión de historias (Lipka y Siegel, 2010). La oralidad es determinante, ya que la lectura en voz alta fue fundamental para la construcción del significado de imágenes. Se demostró que las ilustraciones contribuyen para formar una mente reflexiva (Arizpe, 2005), destacando la importancia de la imaginación en los procesos educativos (Rodari, 2011).

\section{Educación básica}

Los proyectos en educación básica fueron tres, con 78 niños participantes. Así como hablar y escuchar se imitan o aprenden en el entorno inmediato, leer y escribir requieren de un proceso sistemático de enseñanza que se traslada al ámbito educativo (Suárez Muñoz y Suárez Ramírez, 2013). No obstante, en este ámbito se hace énfasis en la utilidad de la lectura. Es decir, la escuela asume que el estudiante alfabetizado puede convertirse en lector por su cuenta, de ahí que se promueva el acto de estudiar, y no tanto la lectura y sus estrategias (Garrido, 2005). Por eso, se debe hacer énfasis en que acercar a los niños a lecturas disfrutables y significativas que estimulen su imaginación, curiosidad y creatividad sentará las bases para el hábito de la lectura.

En tal sentido, los resultados de los proyectos validaron el disfrute con el descubrimiento de palabras, lecturas y autores, por lo que se encontró un aumento en la comprensión de textos, concentración, atención y creatividad. Los niños asumieron de manera natural la escritura para plasmar su visión de lo que los rodea. La lectura en voz alta fue eficaz para inducir a la lectura y para actividades de lectura compartida. Se evidenció que el dominio del lenguaje y la lectura compartida aportan a la adecuada integración al mundo social inmediato, lo que sienta las bases para pensar y resolver problemas, así como expresar emociones y experiencias. Se concluyó que para fomentar la lectura en los niños se deben considerar sus preferencias y propiciar el comentario de textos como método de interpretación, lo que da pauta al descubrimiento de los sentidos de las expresiones (Ballester e Ibarra, 2013).

\section{Profesores de educación básica}

El profesor es el mediador idóneo para acercar las lecturas disfrutables y empáticas a los niños en la educación primaria. El proyecto se realizó con 20 docentes de este nivel. En el diagnóstico los profesores asumieron sus carencias como lectores y sus escasas competencias para realizar esta actividad (Cerrillo, 2010, 2013). En la evaluación sumativa se demostró que transformaron su opinión sobre el significado de leer y aprendieron estrategias sobre la importancia 


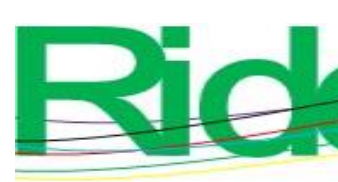

Revista Iberoamericana para la Investigación y el Desarrollo Educativo ISSN 2007 - 7467

de elección de textos considerando el contexto, así como el gran potencial de la biblioteca como coadyuvante en la promoción de la lectura, e hicieron énfasis en la necesidad de un programa permanente de capacitación.

\section{Educación media}

Se realizaron ocho proyectos en instituciones de educación media, con 153 estudiantes y 58 profesores. La lectura y la escritura como actividades complejas requieren en esta etapa un alto sentido de motivación. El promotor de la lectura es puente entre textos y estudiantes, utilizando las mejores estrategias y herramientas de acuerdo con los intereses de los jóvenes. Los resultados establecieron el cambio en el significado de leer y reconocieron la oportunidad que les dio la literatura para situarse en contextos diferentes.

Se observó durante las sesiones un aumento en la participación, discusión y colaboración de los estudiantes, así como el desarrollo de habilidades para concentrarse, deducir, inferir y resumir. La mayoría declaró su interés por seguir realizando lecturas. De hecho, mencionaron que leían alrededor de tres libros en las semanas siguientes al taller. La lectura en voz alta, así como las actividades que vincularon a la lectura con otras artes (como el cine, la música, la pintura, entre otras) fueron señaladas como las prácticas preferidas. Se demostró el aumento de habilidades cognitivas (Garbe, 2005; Larrosa, 2016) en el uso de metáforas, anáforas y resolución de problemas de espacio y abstracción, así como del sentido crítico. Mejoraron expresiones orales, de escritura y la manifestación de emociones. La mayoría expresó que no había tenido un acercamiento previo con la poesía, la cual valoraron muy positivamente después del taller, al igual que las narraciones tradicionales y los textos históricos.

Las recomendaciones finales enfatizaron en más talleres de lectura, así como en mayor acceso a libros de literatura. Se concluyó que la motivación, las actividades lúdicas, las lecturas adecuadas y el contar con contextos sociales de apoyo son fundamentales para promover la lectura entre los jóvenes. Los profesores, a su vez, constataron su responsabilidad como mediadores de la lectura y ampliaron su conocimiento de textos literarios, estrategias y herramientas. Finalmente, plantearon que se debían establecer programas permanentes de capacitación en recursos tecnológicos para el fomento de la lectura. 


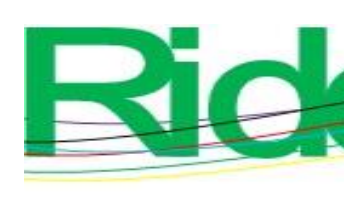

Revista Iberoamericana para la
Investigación y el Desarrollo Educativo
ISSN $2007-7467$

\section{Educación superior}

En este nivel se desarrollaron siete proyectos con grupos de educación superior, con 178 participantes. Se consideró la importancia de afrontar la lectura de textos especializados (Carlino, 2005; Natale, 2013; Stagnaro, 2018), la utilización eficiente de la información (Cassany y Vázquez, 2014; Salado et al., 2017), así como el uso de variados recursos digitales (Cordón García, 2019) para garantizar la lectura significativa de los textos, considerando además las exigencias del discurso de cada área disciplinar (Cordón García, 2019; Gabbiani y Orlando, 2018). Se demostró que esto no ha sido atendido, la mayoría de las veces, en los procesos formativos. Se destacó el promover las competencias lectoescritoras como prácticas transversales que coadyuvan a la multialfabetización utilizando textos literarios. Los proyectos con estudiantes de las áreas técnicas y humanísticas mostraron que es posible construir vínculos entre el discurso literario y las áreas disciplinares. La utilización de las redes sociales propició un acercamiento a la actividad lectora. Los participantes asumieron la importancia de abordar géneros discursivos sobre temas como la homosexualidad y entablar discusiones respetuosas y reflexivas. Descubrieron a la lectura como medio para luchar contra la discriminación, relegación y exclusión. Encontraron un aumento de lecturas que derivó en el incremento de solicitudes de libros de literatura en sus bibliotecas, con lo que se destacó su importancia.

\section{Trabajadores}

Se atendió a cinco grupos de trabajadores universitarios, técnicos, profesionales y bibliotecarios (en total 101 participantes). Se incrementó el conocimiento en el uso de aplicaciones tecnológicas para leer y promover la lectura, y la disposición para usar este conocimiento en sus espacios de trabajo. Los trabajadores dijeron que habían desarrollado competencias de comprensión, reflexión y apropiación de los textos, lo que ayudó al diálogo en sus espacios, así como para compartir lecturas y contrastar opiniones, lo cual redundó en un mejor clima laboral. Los proyectos mostraron cómo los sujetos se apropian de las prácticas sociales en su entorno, es decir, de cómo se aprende al interactuar en determinados grupos (Kalman, 2003). Se demostró cómo se adquieren y utilizan las prácticas comunicativas en entornos específicos. Al respecto, Cassany (2004) explica que "en cada contexto sociocultural, leer y escribir han adoptado prácticas propias, en forma de géneros discursivos que cumplen unas determinadas funciones, con unos roles determinado de lector y autor, con unos usos lingüísticos prefijados y una retórica también preestablecida" (p. 1). Se reafirmó la perspectiva dialógica acentuando la interacción 


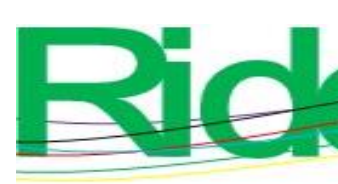

Revista Iberoamericana para la Investigación y el Desarrollo Educativo ISSN $2007-7467$

social y utilizando el lenguaje como vía para adquirir conocimientos y encontrar nuevos significados (Valls, Soler Gallart y Flecha, 2008).

La lectura de literatura con el apoyo de entornos virtuales favorece al diálogo personal en sus contextos, lo que redunda en un mejor desempeño. En algunos casos, como el bibliotecario, establecieron replantear funciones para adoptar estrategias de fomento de la lectura con los usuarios. Esto demuestra que la lectura debe ser abordada como tema transversal y práctica situada y diferenciada, con determinados propósitos, acorde a la comunidad o grupo de que se trate, sin dejar de lado el uso de nuevos discursos, resultado del uso de las TIC (Alonso Arévalo y Cordón García, 2015; Cordón García y Jarvio Fernández, 2015).

\section{Ambientes digitales}

Se realizaron diez proyectos de fomento de la lectura en ambientes digitales que impactaron a 153 participantes directos, a 4148 usuarios de Facebook, a 698 de Instagram y a 800 de Twitter. Estos reportes mostraron lo siguiente: (1) a pesar del uso extendido de los soportes electrónicos de lectura, muy pocos los utilizan para leer literatura o para compartir experiencias lectoras, (2) a la par de la evolución de las TIC, se transforman los mensajes, imágenes, textos y combinaciones entre ellas, así como las nuevas formas de transmisión, y (3) cómo se ha alterado el ecosistema de la lectura y, con ello, cómo se han visto modificadas las expresiones sociales y culturales (Albarello, 2019; Gómez Díaz et al., 2016).

Las estrategias implementadas fueron distintas a las realizadas de forma presencial, para lo que se requieren conocimientos específicos sobre estos medios. La mayoría manifestó que había descubierto comunidades lectoras con las que se propició un diálogo amplio y enriquecedor. Estos soportes permitieron el monitoreo y el acceso a un mayor número de usuarios, así como una integración multidisciplinaria. Los resultados destacaron un aumento en el tiempo dedicado a la lectura de literatura y un mayor número de consumidores. Se corroboró que este tipo de usuarios responden a los procesos de la economía digital, al uso de diversos dispositivos de lectura, de una lectura conectada, lectura social, comentada, compartida en redes sociales, que se enriquece con la escritura online (Alonso Arévalo et al., 2015; Cordón García y Jarvio Fernández, 2015). Fueron evidentes las diferencias en búsqueda de información y en la forma como se expresa la lectura en estos ámbitos. 


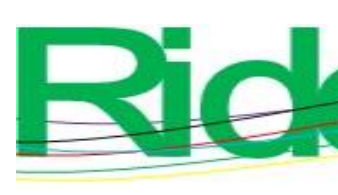

Revista Iberoamericana para la Investigación y el Desarrollo Educativo ISSN 2007-7467

\section{Adultos}

Con adultos se realizaron cuatro proyectos con 95 participantes. Si en la infancia se sientan las bases que propician el gusto por la lectura, en la población adulta existe disposición y motivación para conocer lo que por mucho tiempo les ha sido negado. En México, las personas mayores de 60 años representan $12 \%$ de la población (Inegi, 2019b) y se caracterizan por sus altos índices de analfabetismo (Narro y Moctezuma, 2012); Además, suelen ser una población excluida sobre la cual recaen prejuicios negativos y estereotipos.

La lectura estimula la memoria y evita el deterioro de procesos cognitivos, por ello es imprescindible fomentarla en esta etapa de la vida. En este sector se requiere de estímulos y de textos adecuados al contexto de los participantes. Los resultados mostraron un aumento en el interés por la lectura de literatura, lo que contribuyó al ejercicio de la memoria, a la integración en las actividades, y en algunos casos en la mejora de estados de ánimo. Se favoreció la creación de espacios recreativos como aliciente emocional. Hubo interés por utilizar soportes electrónicos para leer y para establecer vínculos con otros lectores. La mayoría compartió las lecturas con sus familiares.

\section{Grupos marginales o vulnerables}

Se realizaron nueve proyectos con personas marginales o vulnerables (178 participantes). Uno se desarrolló en una colonia de extrema pobreza en la ciudad de Xalapa, Veracruz, con 11 niños y 11 madres; tres en comunidades rurales de alta marginación con 55 niños; uno con cinco amas de casa de colonia marginal; uno en una comunidad de afrodescendientes en el estado de Veracruz (con 20 niños, 10 padres y ocho profesores); uno con un grupo de 14 jóvenes en situación de adicciones; uno con un grupo de 20 mujeres con antecedentes de maltrato, y finalmente uno con un grupo de estudiantes de una escuela de telebachillerato en situación de marginación y violencia.

Los grupos se caracterizaron por la nula actividad previa de prácticas de lectura. Los promotores basaron su proyecto en la transversalidad del acto lector, conocimiento de la problemática y elementos para promover la empatía. Al igual que en los trabajos de Schwartz (2017) y Ceballos (2018), se mostró el impacto de la lectura en situación de vulnerabilidad. Se evidenció el interés por las lecturas y por las actividades realizadas ante la carencia de una oferta cultural para este tipo de grupos. Los padres de familia de niños de sectores marginales mencionaron que habían descubierto lo relevante en incentivar el proceso lector en sus hijos; en 


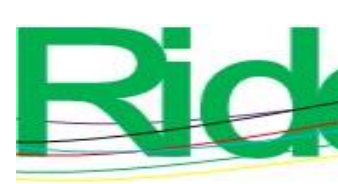

Revista Iberoamericana para la Investigación y el Desarrollo Educativo ISSN 2007 - 7467

padres cambiaron su perspectiva sobre la lectura, y les impactó positivamente el resultado de la práctica lectora en sus hijos; se concluyó que la participación de padres o tutores fue fundamental.

Los jóvenes con discapacidad intelectual ampliaron su manejo de lenguaje, de expresión literaria, de artes visuales y sus herramientas expresivas, principalmente a través de la poesía. Mejoraron habilidades lingüísticas, así como la exploración de sus emociones. Finalmente, al grupo de personas con cáncer, la lectura les ayudó a transitar de un estado de afectación a uno de recuperación. Se concluyó que los libros en los hospitales coadyuvan a una mayor calidad en la estancia, pues propician una adecuada integración y convivencia entre pacientes. Se encontró que el texto literario es un lugar de encuentro, de búsqueda y pérdida, de reconocimiento y ayuda en momentos críticos. Al final del trabajo (en la tabla 1), se muestran algunas expresiones y opiniones recopiladas de todos los proyectos de intervención, a partir de la observación participativa.

El presente estudio indica que para realizar reportes con las características de los 54 utilizados se requiere de agentes promotores capacitados y acciones de vinculación integradas y alineadas al enfoque metodológico explicado.

\section{Conclusiones}

El significado de leer se ha transformado en un acto más complejo, por lo que asumir su prevalencia en una sociedad demanda de la participación de todos los sectores, especialmente el educativo. La preeminencia de lectores básicos contribuye al aumento de brechas de todo tipo, lo cual apremia a que las instituciones educativas transformen y promuevan reformas que consideren programas innovadores para profesionalizar a agentes de cambio que asuman la promoción de la lectura como práctica social situada que coadyuva al dominio de códigos diversos de acuerdo a cada comunidad y contexto. De esta forma se interviene e incide en las prácticas de lectura y escritura en la sociedad, además de fortalecer la vinculación universitaria.

El trabajo muestra la sólida vinculación que se realiza con el entorno, determinada por el compromiso social que muestra la UV, lo que se traduce, primero, en fomentar el hábito de la lectura en todos los grupos y contextos para desarrollar las competencias que orienten a una lectura experta en los diversos formatos y en multiplicidad de discursos, sobre todo en el ámbito educativo. A través de dicha práctica, además, se puede atender a grupos que se han visto relegados o excluidos, pues se puede partir del reconocimiento de que la lectura contribuye al disfrute, a la estimulación de capacidades, al reconocimiento de sentimientos y de intenciones, así como a la promoción de la empatía. 

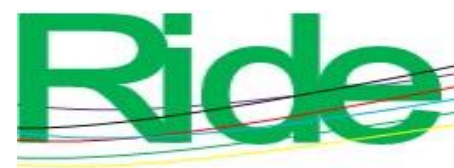

Revista Iberoamericana para la Investigación y el Desarrollo Educativo

ISSN $2007-7467$

En efecto, a través del discurso literario es posible abordar géneros con temas que aborden la discriminación, la relegación y la exclusión. En tal sentido, los grupos atendidos revelan beneficios intelectuales y emocionales, sentido de cercanía gracias a estrategias como la lectura en voz alta y de pertenencia con textos donde se dibujan ellos mismos. Los resultados exponen que estas acciones se traducen en ecos multiplicadores de experiencias con los entornos de muchos de los participantes.

En definitiva, la UV cumple con sus objetivos de pertinencia en la formación profesional, así como con la aplicación innovadora de los saberes para contribuir a la construcción de una sociedad lectora.

\section{Futuras líneas de investigación}

La promoción de la lectura y la escritura se han convertido en temas nodales en el contexto de la educación universitaria, pero ahora se busca que las acciones tengan incidencia en los bajos niveles de lectura en todos los ámbitos de la sociedad. La IES han sido llamadas a asumir la tarea de promover la lectura en diversos grupos y ámbitos de la sociedad, por lo que la vinculación universitaria de debe nutrir con este contenido. Varias instituciones de nuestro país están abordando esta tarea a través de sus programas universitarios de promoción de la lectura. La investigación sobre las técnicas y estrategias, así como sobre los impactos en la sociedad, de este tipo de acciones en los próximos años ocupará las agendas de investigación. Hay temas sustantivos, como los enfoque teóricos, las metodologías de evaluación y la atención a grupos vulnerables que requieren más indagaciones que las realizadas hasta ahora. 


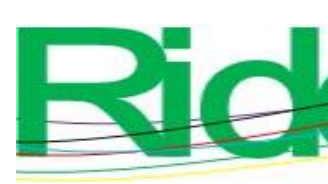

Revista Iberoamericana para la Investigación y el Desarrollo Educativo ISSN $2007-7467$

Tabla 1. Expresiones y opiniones recopiladas a partir de la observación participativa

\begin{tabular}{|c|c|c|}
\hline $\begin{array}{l}\text { Tipo de } \\
\text { proyecto }\end{array}$ & $\begin{array}{c}\text { Perfil de } \\
\text { participante }\end{array}$ & Expresión u opinión \\
\hline \multirow{3}{*}{$\begin{array}{l}\text { Educación } \\
\text { preescolar }\end{array}$} & $\begin{array}{l}\text { Mamá de } \\
\text { niña de } \\
\text { cuatro años }\end{array}$ & $\begin{array}{l}\text { Cada día le gusta leer cuentos y algunas veces ella nos trata } \\
\text { de leer; se ha interesado en la lectura y en escribir el título, } \\
\text { algunas veces pide que se le pongan puntitos para que ella } \\
\text { pueda escribir las palabras que se le dificultan. }\end{array}$ \\
\hline & & $\begin{array}{l}\text { De acuerdo con el ejercicio: regalar tres palabras para crear } \\
\text { una historia (helado, galletas, peinaba), se generó la } \\
\text { siguiente expresión. }\end{array}$ \\
\hline & $\begin{array}{l}\text { Niña de } \\
\text { cuatro años }\end{array}$ & $\begin{array}{l}\text { La abuelita se peinaba su cabello largo al color de la luna, } \\
\text { blancos hilos como de plata, mirando las estrellas junto a la } \\
\text { ventana. La acompañaba su nietecita que era tan graciosa y } \\
\text { comía un helado tan grande como ella. Al terminar se iban a } \\
\text { la cocina a hornear galletas para después comerlas junto a } \\
\text { toda la familia. }\end{array}$ \\
\hline $\begin{array}{c}\text { Educación } \\
\text { básica }\end{array}$ & $\begin{array}{l}\text { Padre de } \\
\text { familia }\end{array}$ & $\begin{array}{l}\text { Una experiencia diferente para los dos, gracias por } \\
\text { enseñarnos a enseñar a nuestros hijos a leer. }\end{array}$ \\
\hline $\begin{array}{l}\text { Educación } \\
\text { media }\end{array}$ & $\begin{array}{l}\text { Estudiante de } \\
13 \text { años }\end{array}$ & $\begin{array}{l}\text { ¡Para mí leer es perderme en un libro que me interesa, y no } \\
\text { saber nada de lo que pasa a mi alrededor! }\end{array}$ \\
\hline \multirow{2}{*}{$\begin{array}{l}\text { Trabajadores } \\
\text { universitarios }\end{array}$} & Bibliotecario & $\begin{array}{l}\text { El conocer bibliotecas digitales será vital en mi trabajo, pues } \\
\text { hay muchos materiales que de ahí se puede extraer para } \\
\text { emplearlos como otra herramienta de apoyo en la promoción } \\
\text { de la lectura. }\end{array}$ \\
\hline & $\begin{array}{l}\text { Trabajador } \\
\text { técnico }\end{array}$ & $\begin{array}{l}\text { Son muy interesantes porque hablan de cosas que le pueden } \\
\text { suceder a cualquiera. Nunca sabes con qué te puedes } \\
\text { encontrar, cada cuento te provoca algo diferente. }\end{array}$ \\
\hline \multirow[t]{2}{*}{$\begin{array}{l}\text { Personas } \\
\text { marginales o } \\
\text { vulnerables }\end{array}$} & Ama de casa & $\begin{array}{l}\text { Sí me gustó mucho que me leyeran en voz alta, fue muy } \\
\text { agradable para mí porque (eh) el escuchar a una persona y } \\
\text { cómo lo (eh) expresabas y cómo lo, lo manejabas fue muy, } \\
\text { agradable porque imaginaba yo más las cosas (eh) ahí } \\
\text { aprendí a leer yo también en voz alta, a tomar entonación, } \\
\text { pausas (este) respiración y el escribir también me agradó } \\
\text { mucho porque me di cuenta la capacidad que tengo para } \\
\text { poder plasmar en un papel lo que yo pienso o sea no es lo } \\
\text { mismo a cuando lo hablo a cuando lo escribo se me dificultó } \\
\text { un poquito pero sí logré que mis ideas se plasmaran en un } \\
\text { papel. }\end{array}$ \\
\hline & Ama de casa & $\begin{array}{l}\text {...pues a mí me gustaría más sobre la vida y obra de Sor } \\
\text { Juana Inés de la Cruz, a mí me atrae mucho Sor Juana Inés } \\
\text { de la Cruz, de Cervantes yo conozco muy poquitito, muy } \\
\text { poquitito, pero a mí sí me gustaría que en un próximo taller } \\
\text { pues nos ampliaras o nos dieran, empezáramos de cero con, } \\
\text { con (este) a leer a Cervantes y, como género en particular }\end{array}$ \\
\hline
\end{tabular}




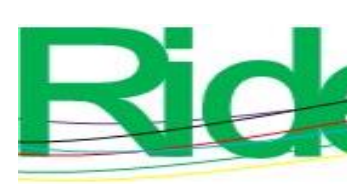

Revista Iberoamericana para la Investigación y el Desarrollo Educativo

ISSN 2007 - 7467

\begin{tabular}{|c|c|l|}
\hline \multirow{1}{*}{} & $\begin{array}{l}\text { fíjate que me gusta lo fantástico, lo último que pues pudimos } \\
\text { leer, fantástico y gótico que, que he estado leyendo fíjate, } \\
\text { como este género por ejemplo Amparo Dávila, Stevenson, } \\
\text { Allan Poe, y Julio Cortázar, mucho, mucho estos me han } \\
\text { atrapado. }\end{array}$ \\
\cline { 2 - 3 } & $\begin{array}{c}\text { Joven en } \\
\text { centro de } \\
\text { adicciones }\end{array}$ & $\begin{array}{l}\text { Gracias por enseñarme que todos tenemos derecho a } \\
\text { sentirnos tristes. }\end{array}$ \\
\hline $\begin{array}{c}\text { Personas con } \\
\text { alguna } \\
\text { discapacidad o } \\
\text { enfermedad }\end{array}$ & $\begin{array}{c}\text { Persona con } \\
\text { discapacidad } \\
\text { visual }\end{array}$ & $\begin{array}{l}\text { No sabía que había autores que hablan de la ceguera y supe } \\
\text { que hay muchos tipos de ceguera: sociales y físicas. }\end{array}$ \\
\hline \multicolumn{2}{|c|}{}
\end{tabular}

Fuente: Elaboración propia

\section{Referencias}

Acosta, S. y Pedraza, E. M. (2019). El texto literario en la universidad: una experiencia para el desarrollo del pensamiento crítico. En Ramírez Leyva, E. M. (coord.), De la lectura académica a la lectura estética (pp. 77-94). UNAM.

Albarello, F. (2019). Lectura transmedia. Leer, escribir, conversar en el ecosistema de pantallas. Ediciones Ampersand.

Alonso Arévalo, J. y Cordón García, J. A. (2015). ¿Para qué servirá la Biblioteca Pública en el futuro? Depende de su capacidad de adaptación a los imparables cambios sociales, $\begin{array}{llll}\text { económicos } & y & \text { tecnológicos. } & \text { Recuperado }\end{array}$ https://gredos.usal.es/bitstream/handle/10366/125206/MiBibliotecaNNTTMB401.pdf;jsessionid=3C8A522409391F98D91B3C205FE14F6C?sequence=1

Alonso Arévalo, J., Gómez Díaz, R. y Cordón García, J.A. (2015). eBooks en bibliotecas: gestión, tratamiento y aplicaciones. Buenos Aires: Alfagrama Ediciones.

Arizpe, E. (2005). El juego de los espacios: los lectores y la interacción entre imagen y palabra en el libro ilustrado infantil. Alter Texto 6(3), 1-36.

Ballester, J. e Ibarra, N. (2013). Comentario de textos. En Martos Núñez, E. y Campos FernándezFigares, M. (coords.), Diccionario de nuevas formas de lectura y escritura (pp. 112-114). Red Internacional de Universidades Lectoras/Santillana.

Bell, S. (2010). Project-Based Learning for the 21st Century: Skills for the Future. The Clearing House, 83(2), 39-43. Doi: http://dx.doi.org/10.1080/00098650903505415

Carlino, P. (2005). Escribir, leer y aprender en la universidad: una introducción a la alfabetización académica. FCE. 


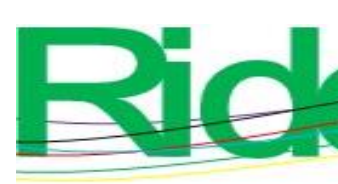

Revista Iberoamericana para la
Investigación y el Desarrollo Educativo
ISSN $2007-7467$

Cassany, D. (2004). Explorando las necesidades de la comprensión. Recuperado de https://repositori.upf.edu/bitstream/handle/10230/21237/Cassany_LyV_2.pdf?sequence=

Cassany, D. (2006). Tras las líneas. Sobre la lectura contemporánea. Anagrama.

Cassany, D. (2012). En línea-leer y escribir en la red. Anagrama.

Cassany, D. y Vázquez, B. (2014). Leer en línea en el aula. Revista Peruana de Investigación Educativa, (6), 63-87.

Castro Azuara, M. C. y Sánchez Camargo, M. (2018). La construcción de opinión: posicionamiento y voz en textos académicos. En Natale, L. y Stagnaro, D. (orgs.), La lectura y la escritura en las disciplinas. Lineamientos para su enseñanza (pp. 137-166). Ediciones UNGS.

Ceballos, J. A. (2018). El rol de la biblioteca pública en la inclusión social del habitante de calle (homeless). Revista de Investigación Bibliotecológica, 32(76), 63-78.

Centro Regional para el Fomento del Libro en América Latina y el Caribe [Cerlalc] (2011). Centro Regional para el Fomento del Libro en América Latina y el Caribe: una historia de libros e integración. CERLALC.

Cerrillo, P. C. (2010). Sobre lectura, literatura y educación. Porrúa.

Cerrillo, P.C. (2013). Mediadores de lectura. En Martos Núñez, E. y Campos Fernández-Figares, M. (coords.), Diccionario de nuevas formas de lectura y escritura (pp. 499-500). Red Internacional de Universidades Lectoras/Santillana.

Cooper, H. M. (2010). Research synthesis and meta-analysis: A step-by-step approach. Sage.

Cordón García, J. A. (2019). La lectura académica en el entorno impreso y digital: contextos, disrupciones y canon. En Ramírez Leyva, E. M. (coord.), De la lectura académica a la lectura estética (pp.17-35). UNAM.

Cordón García, J. A. y Jarvio Fernández, A. O. (2015). ¿Se está transformando la lectura y la escritura en la era digital? Revista Interamericana de Bibliotecología, 38(2), 137-145. Recuperado de https://www.redalyc.org/articulo.oa?id=179038631005

Díaz Barriga, F. y Hernández Rojas, G. (2010). Estrategias docentes para un aprendizaje significativo. Una interpretación constructivista (3. a ed.). México: McGraw Hill.

Escobar, M., Garbarini, M. y López D’Amato, S. (2019). Programas para el fortalecimiento de la lectura y la escritura (PROFLE): Instituto Estudios Iniciales, Universidad Nacional Arturo Jauretche, República Argentina. Revista Argentina de Educación Superior, (19), 64-77. Recuperado de https://dialnet.unirioja.es/servlet/articulo?codigo=7204536 


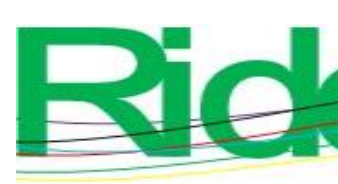

Revista Iberoamericana para la
Investigación y el Desarrollo Educativo
ISSN $2007-7467$

Gabbiani, B. y Orlando, V. (2018). Los textos académicos como géneros discursivos. En Bertolotti, V. (comp.) Lengua, Comunicación e Información (pp. 26-42). Uruguay: Universidad de la República de Uruguay.

Garbe, Ch. (2005). Investigación de la lectura en Alemania. Métodos y resultados. En Ramírez Leyva, E. M. (comp.) Seminario lectura: pasado presente y futuro (pp. 128-159). UNAM. Garrido, F. (2005). La necesidad de entender. Grupo Editorial Norma.

Gómez Díaz, R., García Rodríguez, A., Cordón García, J. A. y Alonso Arévalo, J. (2016). Leyendo entre pantallas. Ediciones Trea.

Instituto Nacional de Estadística y Geografía [Inegi]. (2019a). Estadísticas a propósito del día internacional de las personas de edad. Recuperado de https://www.inegi.org.mx/contenidos/saladeprensa/aproposito/2019/edad2019_Nal.pdf

Instituto Nacional de Estadística y Geografía [Inegi]. (2019b). Población lectora en México con tendencia decreciente en los últimos cinco años. Recuperado de https://www.inegi.org.mx/app/saladeprensa/noticia.html?id=4911)

Jarvio Fernández, A. O. (2019). Lectura académica y lectura de literatura en la Universidad Veracruzana. En Ramírez Leyva, E. (coord.), De la lectura académica a la lectura estética (pp. 139-157). UNAM.

Kalman, J. (2003). El acceso a la cultura escrita: la participación social y la apropiación de conocimientos en eventos cotidianos de lectura y escritura. Revista Mexicana de Investigación Educativa, 8(17). Recuperado de https://www.redalyc.org/pdf/140/14001704.pdf

Larrosa, J. (2016). La experiencia de la lectura. Estudios sobre literatura y formación. FCE.

Lipka, O. and Siegel, L. S. (2010). The improvement of reading skills of L1 and ESL children using a Response to Intervention (RtI) Model. Psicothema, 22(4), 963-969.

Martí, J., Heydrich, M., Rojas, M. y Hernández, A. (2010). Aprendizaje basado en proyectos: una experiencia de innovación docente. Revista Universidad EAFIT, 46(158), 11-21.

Martos Núñez, E. y Campos Fernández-Figares, M. (2013). Diccionario de nuevas formas de lectura y escritura. España: Red Internacional de Universidades Lectoras/Santillana.

Narro Robles, J. y Moctezuma Navarro, D. (2012). Analfabetismo en México: una deuda social. Revista Internacional de Estadística y Geografía, 3(3), 5-17. 


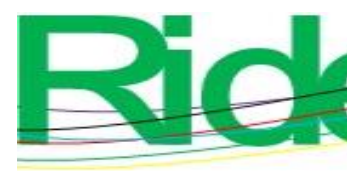

Revista Iberoamericana para la
Investigación y el Desarrollo Educativo
ISSN $2007-7467$

Natale, L. (2013). Integración de enfoques en un programa institucional para el desarrollo de la escritura académica y profesional. Revista Mexicana de Investigación Educativa, 18(58), 685-707.

Ojeda, M. M. y Jarvio Fernández, A. O. (2017). Profesionalización de promotores de lectura con el aprendizaje basado en proyectos mediado por TIC. Revista de Educación a Distancia (54). Doi: http://dx.doi.org/10.6018/red/54/10

Organización para la Cooperación y el Desarrollo Económicos [OCDE]. (2018). Programa para la evaluación internacional de alumnos (PISA). PISA 2018. Resultados. Recuperado de https://www.oecd.org/pisa/publications/PISA2018_CN_MEX_Spanish.pdf?forcedefault= true

Pardo, L. E. (2019). Escritura académica en la universidad: experiencias desde el ensayo académico hasta el artículo académico. En Ramírez Leyva, E. (coord.), De la lectura académica a la lectura estética (pp. 111-124). UNAM.

Pérez, I. G. (2018). Leer en la universidad. En Natale, L. y Stagnaro, D. (orgs.), La lectura y la escritura en las disciplinas. Lineamientos para su enseñanza (pp. 59-112). Ediciones UNGS.

Ramírez Leyva, E. M. (2016). De la promoción de la lectura por placer a la formación integral de lectores. Revista de Investigación Bibliotecológica. Archivonomía, Bibliotecología e Información, 30(69). 95-120.

Rodari, G. (2011). Gramática de la fantasía. Planeta

Rodríguez, W. C. (2013). El lugar de la afectividad en la psicología de Vygotski: reflexividad histórica y reivindicación. Propósitos y Representaciones, 1(2), 105-129. Doi: http://dx.doi.org/10.20511/pyr2013.v1n2.35

Romero, E. S. y Barboza, J. L. (2012). Estrategias interactivas e investigación acción para consolidar la comprensión de la lectura. Multiciencias, 12(1), 64-71.

Rosing, T. K., Rettenmaier, M., De Cassia Tussi, R. y Segal, B. (2013). Inclusión. En Martos, E. Núñez y Campos Fernández-Figares, M. (coords), Diccionario de nuevas formas de lectura y escritura (pp. 314-317). Red Internacional de Universidades Lectoras/Santillana.

Salado Rodríguez, L. I., Ramírez-Martinell, A. y Ochoa Landín, R. I. O. (2017). Hábitos de lectura y afinidad tecnológica de los estudiantes universitarios: estudio comparativo de cinco

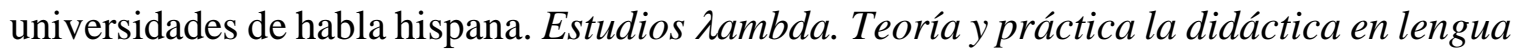
y literatura, 2(1), 1-24. 
